\title{
DIVERSE TIME-FREQUENCY DISTRIBUTIONS INTEGRATED TO AN ART2 NETWORK FOR NON-DESTRUCTIVE TESTING
}

\author{
Benítez-Pérez H., ${ }^{1}$ Medina-Gómez L. ${ }^{2}$ \\ ${ }^{2}$ Departamento de Física, Facultad de Ciencias, UNAM, Apdo. Postal 20-726 \\ ${ }^{1}$ Departamento de Ingeniería de Sistemas Computacionales y Automatización, IIMAS, UNAM, \\ Apdo. Postal 20-726, Del. A. Obregón, México D. F., CP. 01000, México. \\ Fax: ++52 5556160176 , Tel: $\left(^{*}\right)++525556223623$ \\ Email: $\left(^{*}\right)$ hector@uxdea4.iimas.unam.mx
}

\section{ABSTRACT}

The use of several techniques for non-destructive testing is a common strategy for detecting and classifying flaws in aluminium material. Techniques like multiresolution data analysis and data classifiers are valuable for obtaining as much information as possible from the flaws. The combination of both techniques allows the clear definition of several characteristics like localization, size and form. In this study, localization using Time-Frequency Distribution feature extraction and an ART2 neural network as a classifier is the main goal.

KEY WORDS: Non-destructive Testing, Neural Networks, Time Frequency Distribution Approach

\section{INTRODUCTION}

The use of Ultrasonic Non-Destructive Evaluation presents a suitable scanning procedure for different materials. This technique consists of a pulse-echo system integrated in a single transducer. The receiving transducer is excited by scattered waves, and a transient change appears across the transducer faces and generates an electrical pulse in the receiver section (Lester et al., 1998). The transducer is moved along a linear scan path where the temporal distance of a particular flaw, the bottom part of the material, and the inherent grain thickness are monitored. This strategy is known as Bscan display.

The aim of this paper is to detect flaws using ART2 networks and Time-Frequency Distribution approaches. In order to process this information, the use of pattern recognition for signal processing presents a novel approach to determine temporal distance based upon echoes analysis, without using classical signal processing techniques. The use of model building instead of data acquisition is a significant step, which involves characterization and abstraction of the process (Legendre et al., 2001). Flaws are detected within a material by pulse echoes using ultrasonic measurements. This approach consists of processing a signal using time-frequency distribution and ART2 neural network (Lester et al., 1998 and Kirby, 2001). In particular, Time-Frequency Distribution such as Wigner-Ville, Choi-Williams, and Bessel (Martin et al., 1985; Garcia-Nocetti et al., 2001) presents a feasible approach. Moreover, this study classifies the response of different levels of this type of Time-Frequency Distribution under the evaluation of a sample of aluminium material. It uses the speed of sound elapsed in a time difference between two reflections within the same propagation medium. Those echoes may represent the type of grain, thickness of the material, and different sorts of flaws within the material.

In order to determine pattern behaviour of temporal distance, the use of neural networks is pursued. Different strategies have been proposed up to this stage. Vachtsevanos et al. (2001) present a wavelet neural network based upon Radial Basis Function Neural Network and basic cost rap wavelet 
decomposition in order to extract different features related to flaw presence. Fang et al. (2000) present the use of a more efficient orthogonal-neural network based on the behaviour of scaling function and the corresponding mother wavelet, referred to as orthogonal wavelet neural network. Moreover, Ciftcioglu (1999) shows an analysis from neural networks to wavelet networks by means of a neural network as a multivariate function approximation. Yu et al. (1996) built the wavelet decomposition by using a B-Spline as the scaling function. Alternatively, Tang et al. (2000) present a dynamic neural network with the hidden layer that consists of wavelets for non-linear system identification. In the study, the use of autoregressive connection is introduced into wavelet based neural network. Angrisani et al. (2001) and Solis et al. (2001) present a combination of wavelet decomposition and artificial neural networks that combine analysis of non-stationary signals and classification abilities. Related techniques to nondestructive evaluation have been presented by Demirli et al. (2001a,b). Similar work to achieve flaw localization and characterization has been developed previously, as presented by Moisen et al. (2004), Benitez-Perez et al. (2002) and Solís et al. (2002) using diverse neural networks and multiresolution wavelets.

In terms of flaw detection, the strategy proposed here is named Flaw Classification and Localization. In fact, this flaw classification and localization enhances the availability of the system to localize abnormal behaviour rather than enhancing the safety of the system. Different approaches have been followed by the use of neural networks, Principal Component Analysis (PCA) (Moya et al., 2001), and statistical approaches.

The approach taken in the current study is based upon on-line pattern recognition cluster classification techniques (Baraldi et al., 1999) which are enhanced by Time Frequency Distribution (TFD) as preprocessing module of pulse-echo signal. The resultant winning pattern vector shows which TFD level presents the echo pulse without disturbances. Therefore, the associated weight vector represents the response of this decomposition.

Following this brief description, this paper presents a review of these Time-Frequency Distribution algorithms used in this work, the ART2 network approach, the current approach based upon these TFD algorithms and ART2 networks, and preliminary results.

\section{TIME-FREQUENCY DISTRIBUTION (TFD)}

Several types of Time-Frequency Distributions are based upon the Cohen proposal (Cohen, 1989, Forberg et al., 1999), such as Choi-Williams, Bessel, Wigner-Ville or Born Jordan, among others. In this work, three distributions are described: Wigner-Ville, Bessel, and Choi-Williams, as each defines particular characteristics suitable for flaw detection. Wigner-Ville TFD is used to estimate the mean frequency quasi-instantaneous of current echo-pulse signal. This TFD is used due to its computational simplicity (Garcia-Nocetti et al., 2001). The goal of TFD is to represent a signal based upon its frequency and power components by a structured decomposition.

Time-Frequency Distributions of Cohen class can be represented by (Cohen, 1989)

$$
C(t, \omega)=\frac{1}{4 \pi^{2}} \iiint x^{*}\left(u-\frac{1}{2} \tau\right) x\left(u+\frac{1}{2} \tau\right) \phi(\theta, \tau) e^{-i \theta t-i \tau \omega+i \theta u} d u d \tau d \theta
$$

where $\omega$ is the angular frequency, $x(t)$ is the analytical signal and $x^{\star}(t)$ its complex conjugate, and $\phi(\theta, \tau)$ is a two dimensional kernel, in the correlation plane $\theta-\tau$, that determines the distribution and its properties. The analytic signal is defined as a complex time function having a Fourier transform that vanishes for negative frequencies (Oppenheim et al., 1999 and Mitra, 2001).

The kernels and TFD equations of Wigner-Ville, Bessel and Choi-Williams's Time-Frequency Distributions are defined in Table 1.1. 
Diverse Time-Frequency Distributions Integrated to an ART2 Network for Non-Destructive Testing, Benítez-Pérez H. et al, 14-32

\begin{tabular}{|l|c|c|}
\hline \multicolumn{1}{|c|}{ TFD } & $\phi(\theta, \tau)$ & Time Frequency Representation \\
\hline Wigner-Ville & 1 & $V W D(t, \omega)=\int_{-\infty}^{+\infty} x\left(t+\frac{\tau}{2}\right) x^{*}\left(t-\frac{\tau}{2}\right) e^{-i \omega \tau} d \tau$ \\
\hline Bessel & $\frac{J_{1}(2 \pi \alpha \theta \tau)}{\pi \alpha \theta \tau}$ & $B D(t, \omega)=\int_{-\infty}^{+\infty} \frac{2}{\pi \alpha \tau} \int_{-\infty}^{+\infty} \sqrt{1-\left(\frac{t-u}{\alpha \tau}\right)^{2}} U_{0}\left(\frac{t-u}{\alpha \tau}\right) x\left(u+\frac{\tau}{2}\right) x^{*}\left(u-\frac{\tau}{2}\right) e^{-i \omega \tau} d u d \tau$ \\
\hline Choi- & $e^{i \frac{\theta \tau}{2}}$ & $C W D(t, f)=\int_{-\infty}^{+\infty} \sqrt{\frac{1}{4 \pi \tau^{2} / \sigma} \int_{-\infty}^{+\infty} e^{-\frac{(t-\mu)^{2}}{4 \tau^{2} / \sigma}} x\left(\mu+\frac{\tau}{2}\right) x^{*}\left(\mu-\frac{\tau}{2}\right) d \mu e^{-j 2 \pi f \tau} d \tau}$ \\
Williams & & \\
\hline
\end{tabular}

Table 1.1 Time frequency kernels and their continuous form representation.

Since the acquired experimental signals are real, discrete and finite, the time frequency distribution has to be represented as a windowed discrete version of the time frequency representations defined in Table 1.1.

The windowed Wigner-Ville Time-Frequency Distribution in a discrete form is expressed as

$$
D W V D(n, k)=2 \sum_{\tau=-N+1}^{N-1} W(\tau) W^{*}(-\tau) e^{-i \frac{2 \pi k \tau}{N}} x(n+\tau) x^{*}(n-\tau)
$$

where the index $-N+1 \leq n \leq N-1$ represents the discrete time; $0 \leq k \leq N-1$ represents frequency vector; $W(n)$ is a sampled time window (Hanning), and vector $x(n)$ is the analytical signal of the measured signal.

The Bessel TFD in a discrete from can be expressed as

$$
D B D(n, k)=2 \sum_{\tau=-N+1}^{N-1}\left(W(\tau) W *(-\tau) e^{-j 2 \pi k \tau}\right) * \sum_{\mu=-2 \alpha|\tau|}^{2 \alpha|\tau|}\left(\frac{1}{\pi \alpha \tau} \sqrt{1-\left(\frac{\mu}{2 \alpha \tau}\right)^{2}} x(\mu+n+\tau) x *(\mu+n-\tau)\right)
$$

In a similar manner, Choi-Williams distribution in a discrete form is presented.

$$
D C W D(n, k)=2 \sum_{\tau=-N+1}^{\tau=N+1} W(\tau) e^{\left(\frac{-i 2 \pi k \tau}{N}\right)}\left[\sum_{\mu=-2 \alpha \tau}^{2 \alpha \tau}\left(\frac{1}{\left(\frac{4 \pi \tau^{2}}{\sigma}\right)} e^{\left(\begin{array}{c}
(\mu+\tau / 2)^{2} \\
4 \tau^{2} / \sigma
\end{array}\right)} x(\mu+\tau+n) x^{*}(\mu+n-\tau)\right]\right]
$$

It is important to note that index $\mu$ produces the correlation over the analytical signal $x(n)$, with respect to $\mu+n+\tau$ window length. For the last two cases, there is a constant value named $\alpha$ that is related to 
the variance of the data, $\sigma$ is the time division per frequency. A complete review of this technique can be found in Cohen (1989).

\section{ART2 NEURAL NETWORK}

This neural network has been proposed since it is a powerful classifier that can be adapted fairly quickly (Carpenter et al., 1996), when a new pattern is presented, preprocessing procedure extracts enough information to be classified without glitches. Other neural networks can be used, however, the computational cost is increased exponentially and no additional advantage is obtained in comparison to ART2A.

Having defined the group of TFDs, a review of the neural network algorithm is given here. Adaptive Resonance Theory (ART) network was originally proposed by Carpenter et al., (1996). This network works as a pattern classification non-supervised network. The objective of this technique is to define certain groups (from actual data) around specific data points named as cluster centres. When a new group appears, its centre is identified in order to be defined as a cluster (Whiteley et al., 1996). This new centre works as an identifier of this group. The output of the fuzzy system shows the presence of this new cluster as a new combination of values (zero and one).

The ART2 network has been implemented following the approach presented by Frank et al., (1998), shown in Fig. 3.1. The idea is to identify already-classified material patterns and categorize new temporal distances based upon the classification of new patterns. The use of a new group of patterns does not overcome the identification of the physical meaning of the new classified pattern. This work still should be performed off-line by the expert.

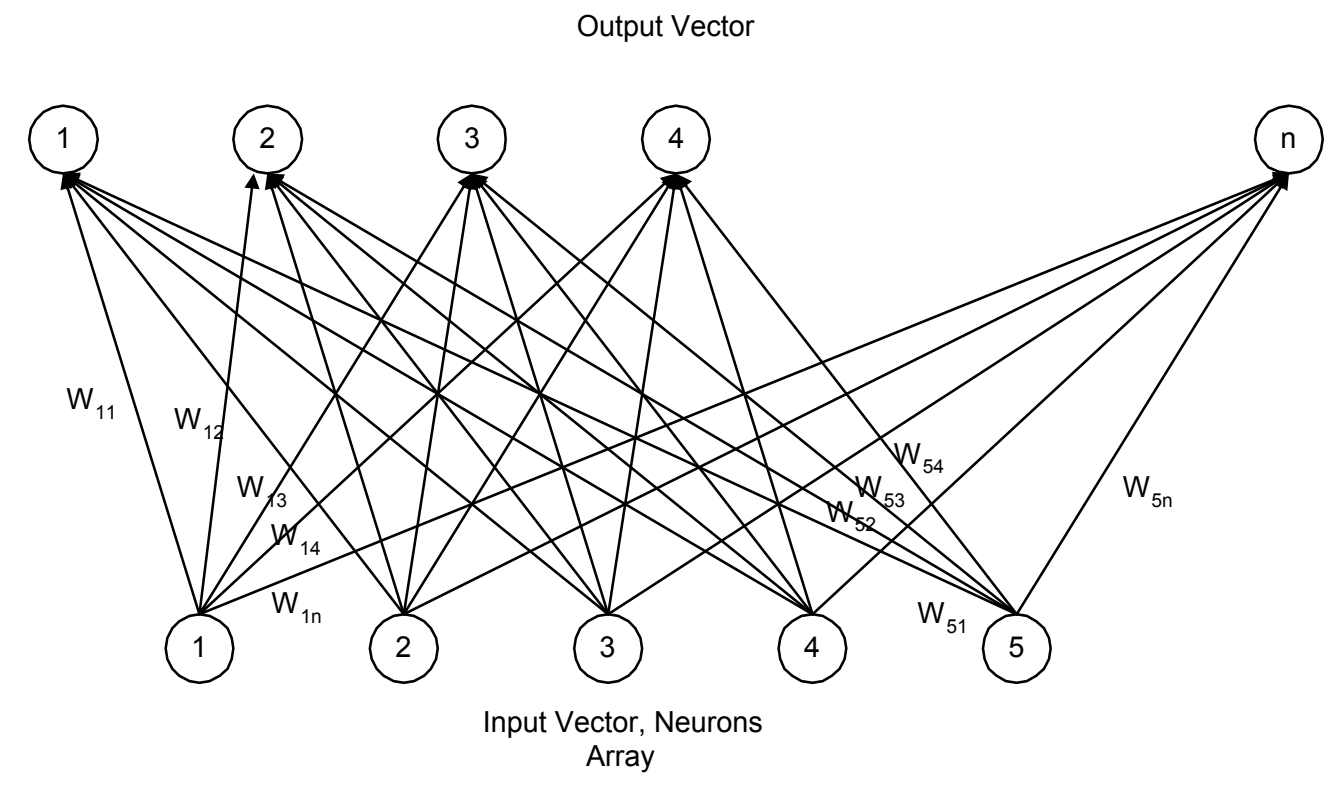

Figure 3.1. Typical ART2 network

The current input of the network is stated as vector A. This is normalized using the Euclidian media shown in eqn. 5 . 


$$
\left.I=\frac{A}{\| A \mid}=\frac{A}{\sqrt{\sum_{i=1}^{m} a_{i}^{2}}} a_{i} \geq 0 \quad \forall i \quad\|A\|\right\rangle 0
$$

where $m$ is the number of elements from non-normalized input vector $A$. The new generated vector $I$ is used to perform another vector named as $t$ based on eqn. 6 .

$$
t_{j}=\sum_{i=1}^{m} w_{i j} i_{i}
$$

where $w_{i j}$ is an element of weight matrix. This matrix is generated by previous pattern classification. From $t_{j}$ element, a new matrix is performed stated as $T$, which represents the interaction between the already known weight matrix and the input vector. The maximum element from current $t$ vector becomes the winner for this input vector. Having defined this interaction, the stage bottom-up is completed. The maximum value of current $t$ vector is compared against the vigilance parameter, $\rho$, in order to determine if this current minimum value is close enough to the vigilance parameter. If so, the related winning $W_{j}$ vector is declared as a representative pattern of this input vector. The winning $W_{j}$ vector is modified following eqn. 7 .

where $\eta$ is defined as a learning parameter.

Alternatively, if a comparison between current maximum $t_{j}$ element and the vigilance parameter is not enough, a new pattern has been identified. Then, a new $W_{j}$ vector is concatenated to the weight matrix. This new vector is the current input vector $I$.

\section{THE PROPOSED ALGORITHM}

Having reviewed both strategies used in this paper, it is necessary to define the proposed algorithm. This is a combination of two techniques in a cascade mode (Fig. 4.1). The algorithm has two stages, and the first is a learning stage where the ART2 network is trained using several input vectors that are decomposed through the TFD procedure. Each vector produces a decomposed matrix processed by the ART2 network, which classifies each vector from the decomposed matrix. This procedure is performed until all selected vectors have been processed.

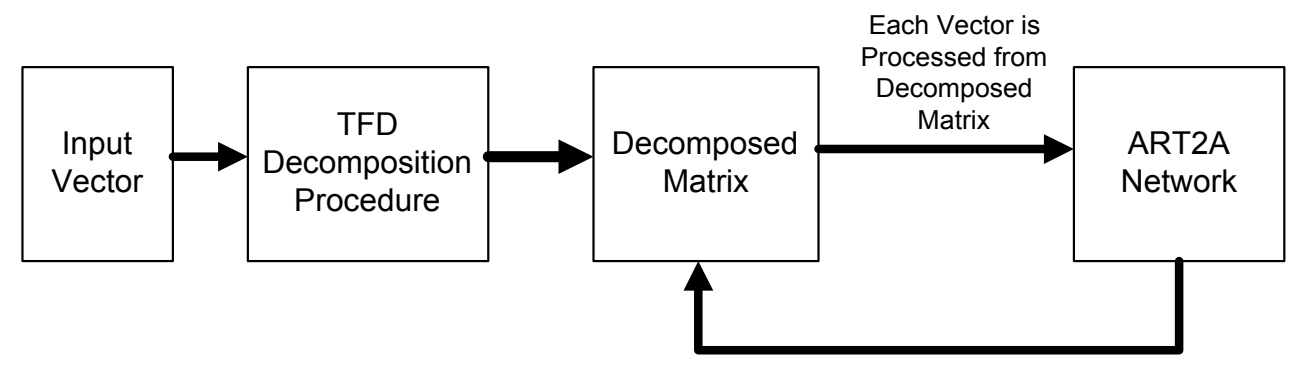

Figure 4.1 Schematic procedure of learning stages from the proposed algorithm

The second stage is a classification stage in which the ART2 network performs classification without the use of "TFD Decomposition Procedure". Eventually, if none of the current patterns are declared winners, the learning stage is used for those un-committed prototypes. Fig. 4.2 shows the implementation of the second stage (Classification Stage), considering the eventual incorporation of the learning stage. 


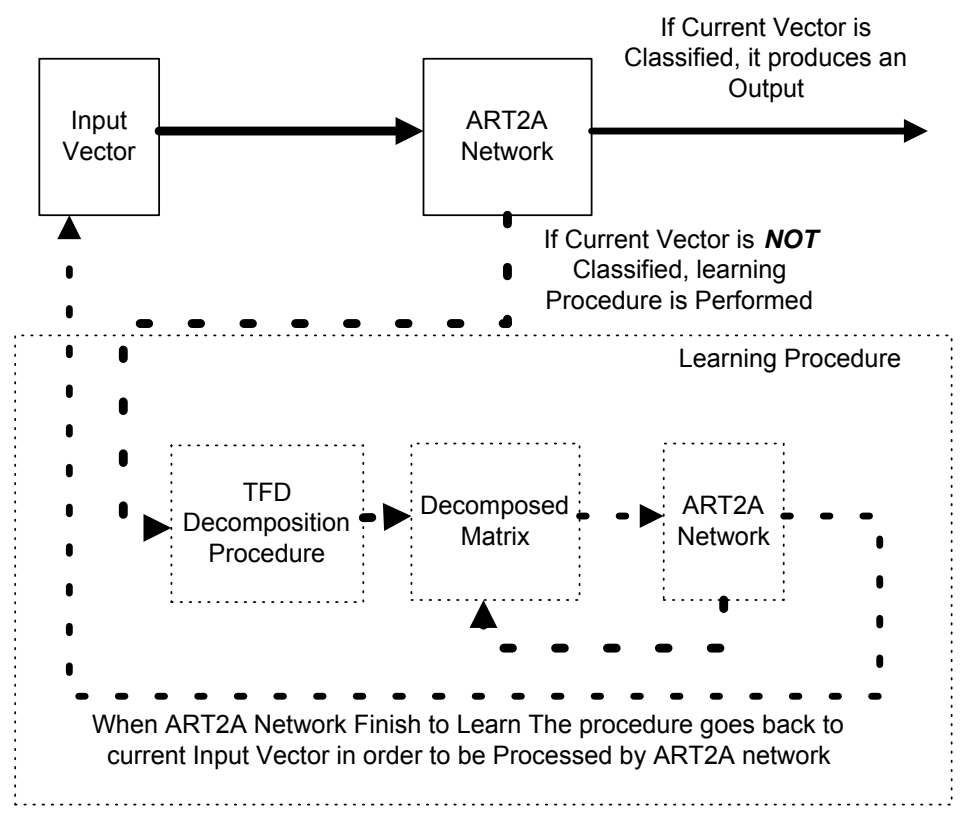

Figure 4.2 Schematic procedure of classification stages from the proposed algorithm

The advantage of the second stage is in terms of non-processing the "TFD Decomposition Procedure" due to those already known patterns. The impact of this second stage is in terms of computing cost. A review of how this proposed algorithm is used for Non-Destructive Testing is presented in the next sections.

\section{CURRENT APPROACH}

The approach proposed in current study is described by a case study that is concerned with the evaluation of an aluminium sample material. Previous work related to the definition of this approach has been presented by Solis et al. (2001). The schematic diagram to evaluate materials is shown in Fig. 5.1. Fig. 5.2 shows the block diagram of the setup experiment where the movement of the transducer is lateral and the inspected material is within the water tank. The proposed strategy has been developed under MATLAB using Time Frequency Distribution and ART2 network. The result of the generic Time Frequency Distribution approach is a matrix in which the elements are the energy associated to a specific time and frequency pair. This matrix is processed by ART2 network in order to classify a finite number of patterns.

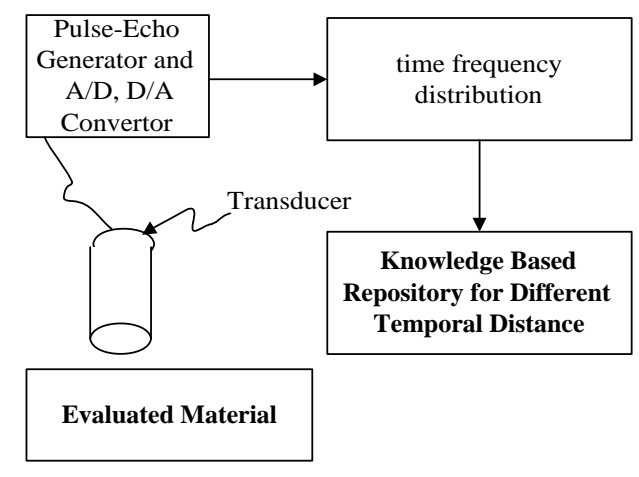

Figure 5.1 TFD-ART2 Approach 


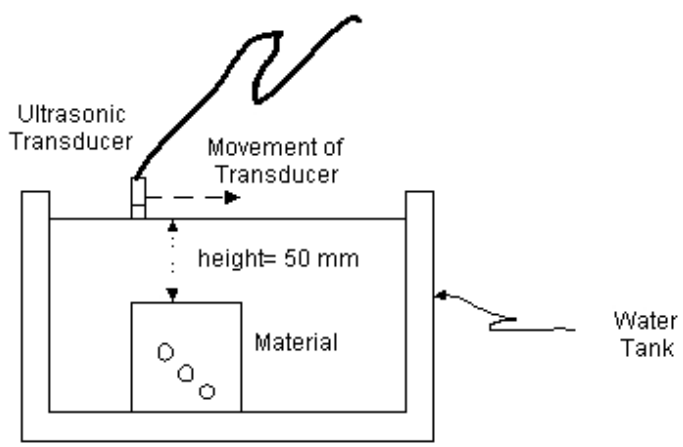

Figure 5.2 Schematic diagram of setup experiment

From this pattern matrix, different features of the same material can be evaluated without the TFD technique. The evaluation of the sample material is performed in one axis, which is named "face one". During this evaluation, if there is a flaw without a related pattern, this is processed using Time Frequency Distribution such as Wigner-Ville or any other in order to generate a decomposed matrix. A new pattern is declared by ART2 network as soon as this is processed using the decomposed matrix.

The information presented as input of this approach is the normalized sample of the signal obtained from the pulse echo generator (Fig. 5.2). The flow chart shown in Fig. 5.3 depicts these two stages: (1) the training procedure using Time Frequency Distribution and neural network processes, and (2) named as classification procedure without using the Time Frequency Distribution process.

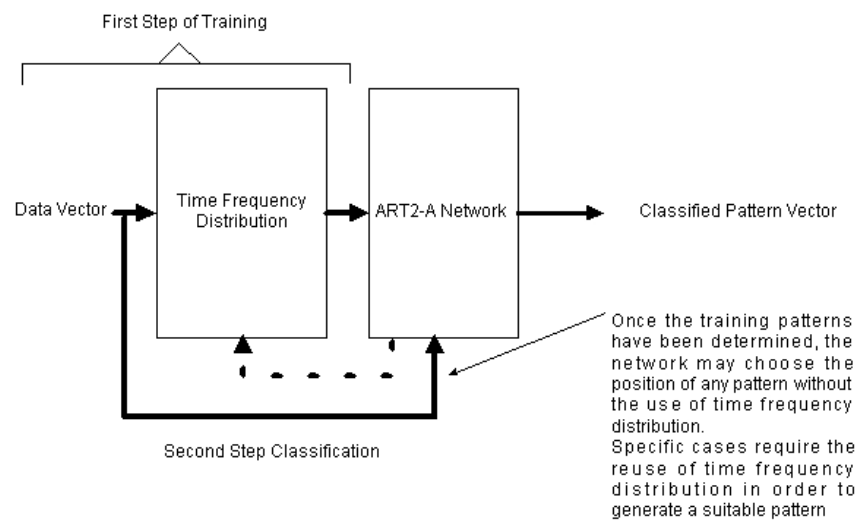

Figure 5.3 Training and classification processes

Following training and classification strategies, it is possible to determine which temporal distance (out of the knowledge database) is the most suitable and represents the evaluated section of the material without using the Time Frequency Distribution process. The temporal distance is represented by the patterns database.

This approach is used to B-Scan an aluminium material in order to make a pattern database of different characteristics with several flaws and borders of the material named as scenarios. Each sample is captured and discretized by an oscilloscope; it is passed to the MATLAB environment in order to be processed. Each sample depends on each step from the linear movement of the transducer. Each step along the B-scan has a distance of $0.635 \mathrm{~mm}$. The sampled material is made of aluminium with a volume of $7 \times 7 \times 4 \mathrm{~cm}$, as shown in Fig. 5.4 . 


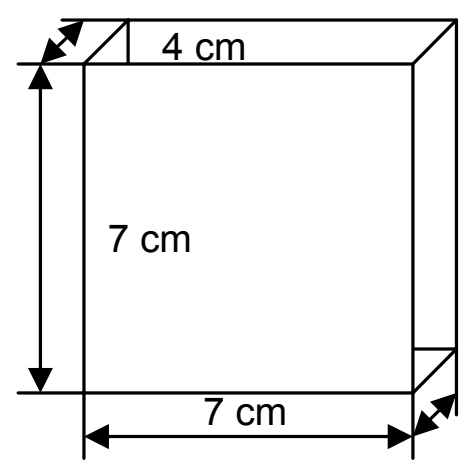

Figure 5.4 Sampled material

The sampled material is composed of $90 \%$ aluminium and has (Fig. 5.5.a) three determined flaws of 2$\mathrm{cm}$ depth and a diameter of $0.5 \mathrm{~cm}$ (flaw1, flaw 2 and flaw 3). Positions of these flaws are shown in Fig. 5.5.b.

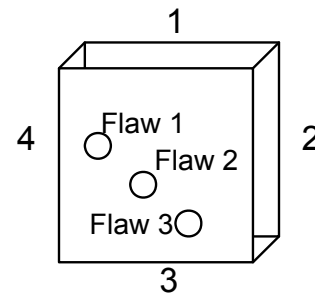

Fig. 5.5.a

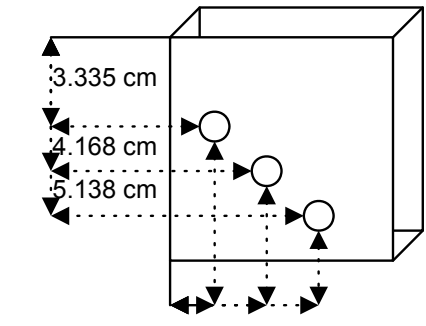

Fig. 5.5.b

Figure 5.5 Sampled material with flaws

\section{CURRENT APPROACH}

Preliminary results show how this approach performs to generate a B-scan pattern database. In order to determine the temporal distance of the flaws in the sampled material, the following experimental setup was used:

- Oscilloscope MATEC 25msamples/second. Time base of 20 microseconds.

- $\quad$ SR-9000 MATEC card with a $22.5 \mathrm{db}$ gain no damp.

- Krautkrammer transducer with a central frequency of 3.5 Mhz.

- Lab Scanner (water tank) trademark MATEC.

In order to evaluate the aluminium material without using the Time Frequency Distribution approach, it is necessary to normalize each sample from a range of values between 0 and 1 (Eq. 5). Furthermore, the length of the input vector (captured through the transducer) consists of 1000 points, whereas just 499 points are used in the TFD approach. Moreover, neural network learning and vigilance parameters are set to 0.85 and 0.3 , respectively. The number of neurons is 50 at the second layer and the size of the neural network is 260 neurons. 


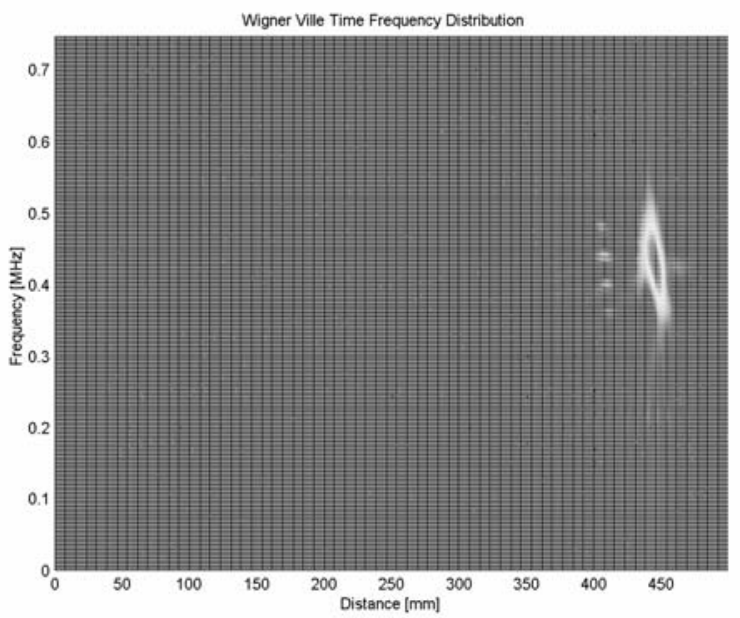

Figure 6.1 Echo-pulse signal processed using TFD without flaw

Fig. 6.1 shows the result of an echo-pulse signal processed by Wigner-Ville TFD. In this case, a matrix of 499x350 elements has been formed. The y-axis (Frequency) represents those frequencies selected during Time Frequency Distribution. This graphic shows the bottom part of the material at the main frequency of the chosen transducer.

The $x$-axis (Distance) is the temporal distance of the echo-signal. It is essential to note that this distribution is centred with respect to the main frequency of the transducer. The neural network processes this matrix in order to generate a number of representative patterns.

Thereafter, Fig. 6.2 shows the result of another echo-pulse signal where the first flaw is shown, as well as the bottom part of the material. The main frequency presents the location of this flaw. In this case, the temporal distance shown between the first flaw and the bottom of the sampled material is the actual distance among them.

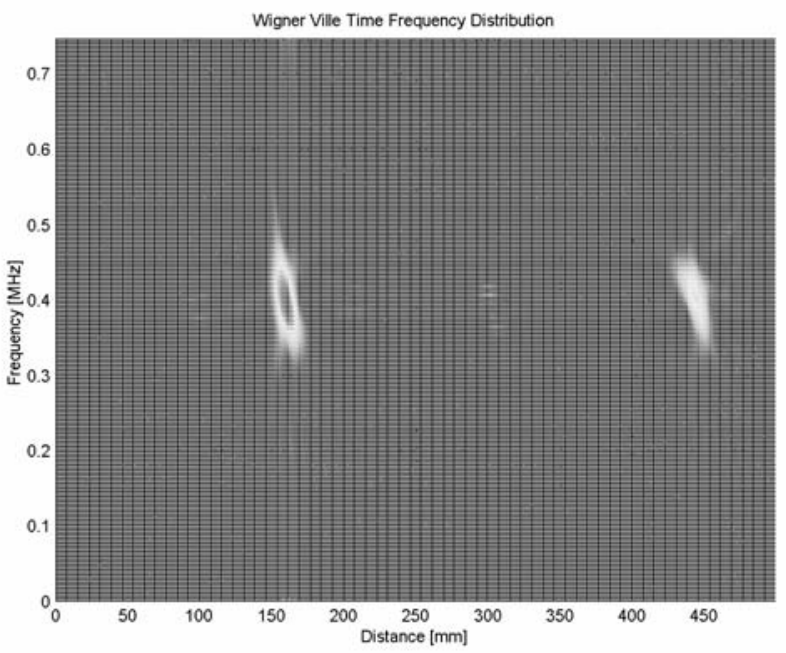

Figure 6.2 Echo-pulse signal processed using TFD with first flaw

Figs. 6.3 and 6.4 present similar information with respect to flaw 2 and flaw 3 , respectively. However, both present a small "spot" of a flaw between the bottom and the current flaw. This "spot" is due to inherent crossing terms within Wigner-Ville TFD. 


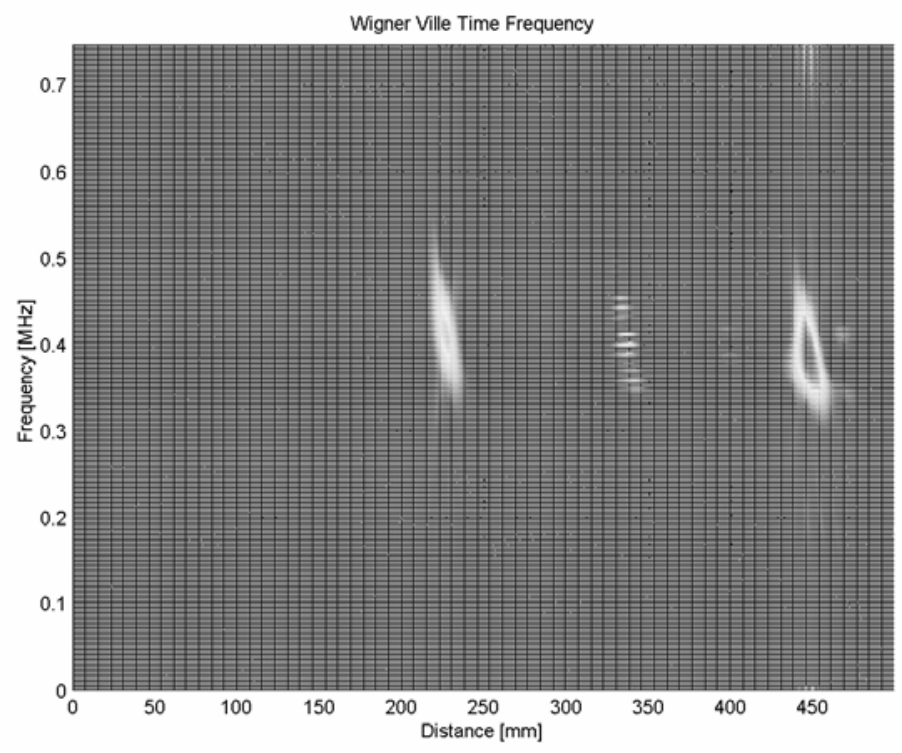

Figure 6.3 Echo-pulse signal processed using TFD with second flaw

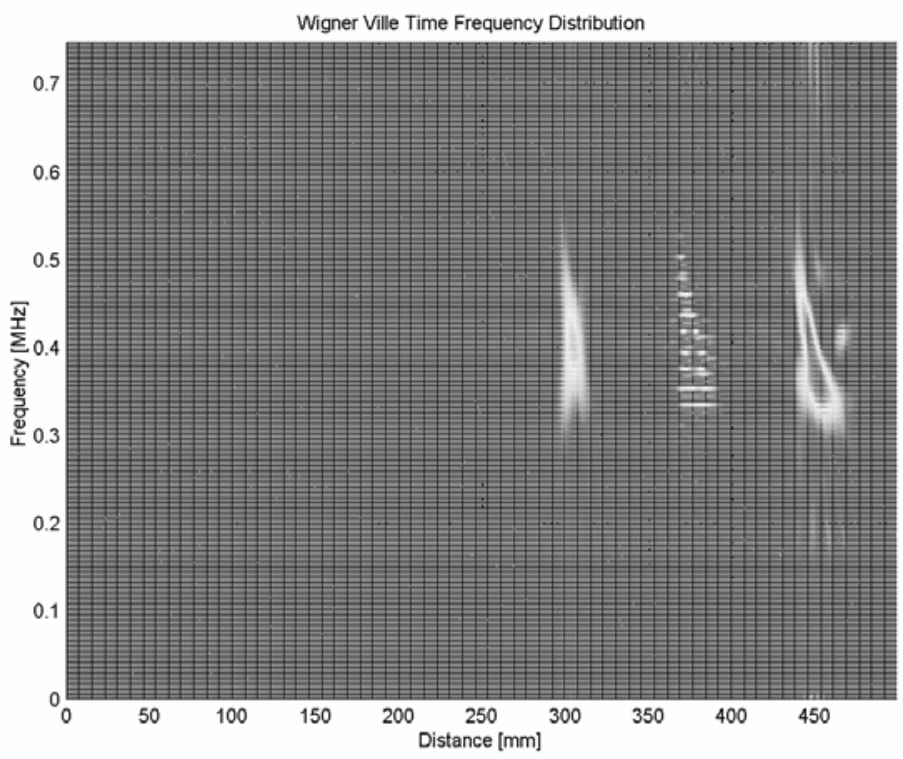

Figure 6.4 Echo-pulse signal processed using TFD with third flaw

The ART2 network processes ten of these matrices in order to generate a confident pattern database, which characterized the sampled material. The total number of sample echo-pulse signals obtained along the path is 100 .

Having trained the network with those selected signals and their respectivematrices, a transversal view of sampled material is constructed based upon the weight matrix of the ART2 network. Fig. 6.5 depicts this information, where the $y$-axis presents the number of patterns and the $x$-axis presents the temporal distance in terms of points. 


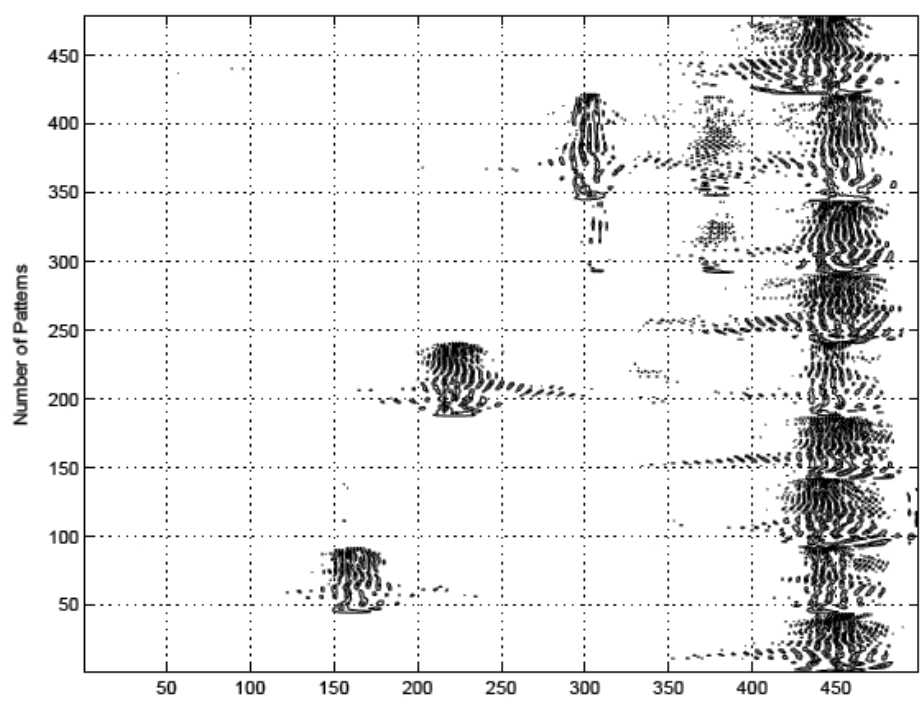

Figure 6.5 Transversal view of whole sampled material based upon the ART2 network approach

Fig. 6.6 shows one signal used to test the ART2 network without having been processed by the TFD module. In this case, one of the patterns (no. 47) has been selected. The number of patterns remains constant (130).
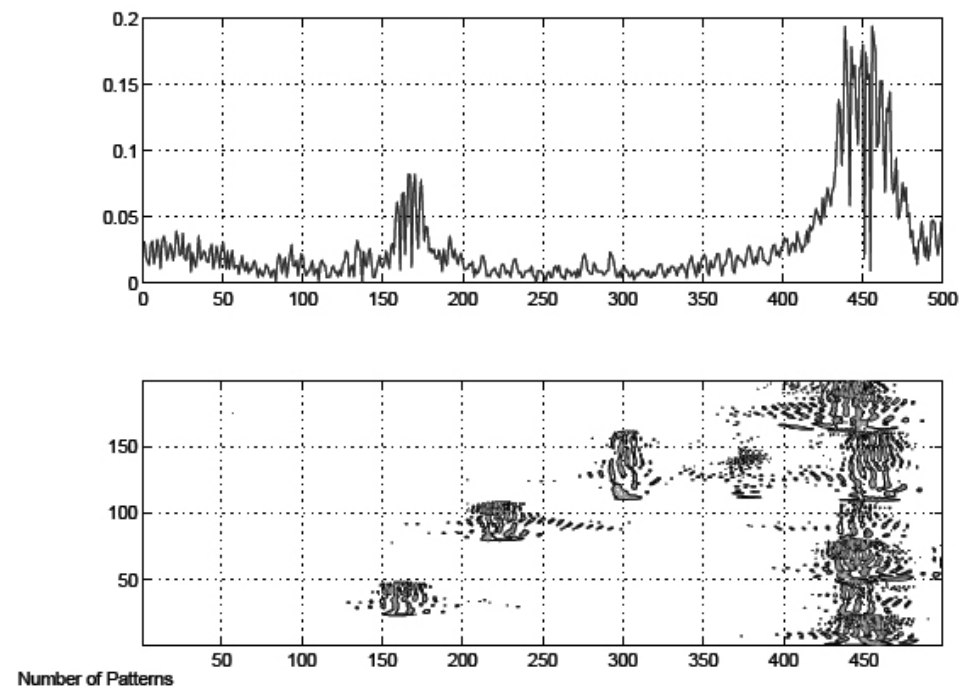

Figure 6.6 Evaluated database based upon another pulse-echo signal with same flaw at different scan position

Having explored the response of Wigner-Ville TFD in combination with the ART2 network, the next TFD (Bessel) is reviewed. The following group of results show its performance. First, the Bessel TFD of the processed signals are shown in Figs. 6.7, 6.8, 6.9, and 6.10. Next, global results processed by BesselART2 algorithm generating the B-scan pattern database are shown in Figs. 6.11 and 6.12.

It is necessary to normalize each sample from a range of values between 0 and 1 , as done for the Wigner-Ville approach. Furthermore, the length of each vector consists of 1000 points, but only 499 of them are actually taken into account. Moreover, neural network learning and vigilance parameters are 
set to 0.92 and 0.3 , respectively. In this case, these parameters have changed in order to obtain the best possible response.

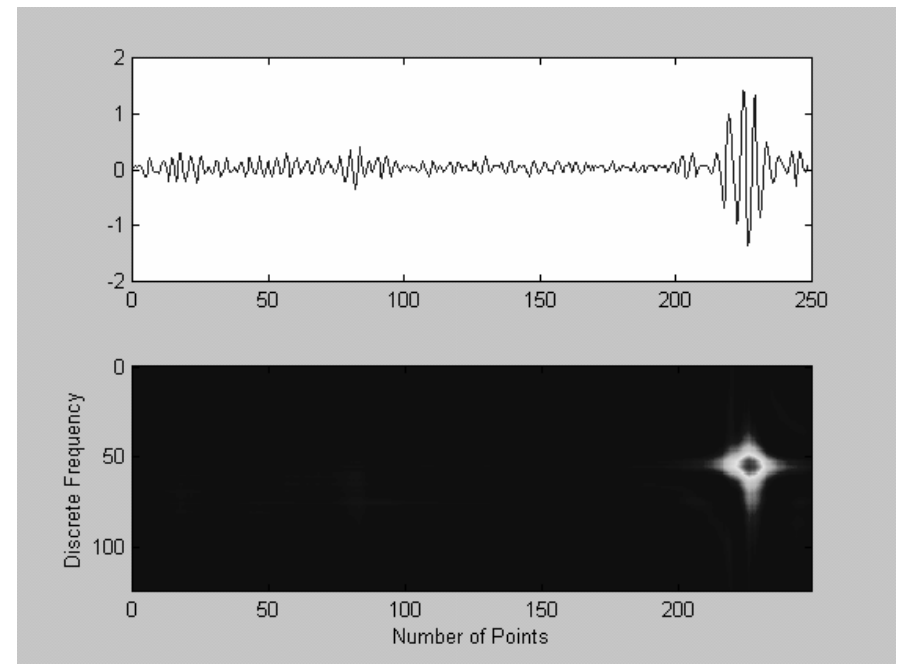

Figure 6.7 Echo-pulse signal processed using TFD without flaw

Fig. 6.7 shows the result of an echo-pulse signal that has been processed by Bessel TFD. The y-axis shows the frequencies, and the x-axis represents the temporal distance. The selected echo-pulse signal and TFD response show the bottom of the tested material. It is essential to note that this distribution is centred with respect to the main frequency from the transducer. The neural network processes this matrix in order to generate a number of representative patterns. Thereafter, Fig. 6.8 shows the result of another echo-pulse signal, where the first flaw is shown. The frequency associated with the transducer presents the location of this flaw.

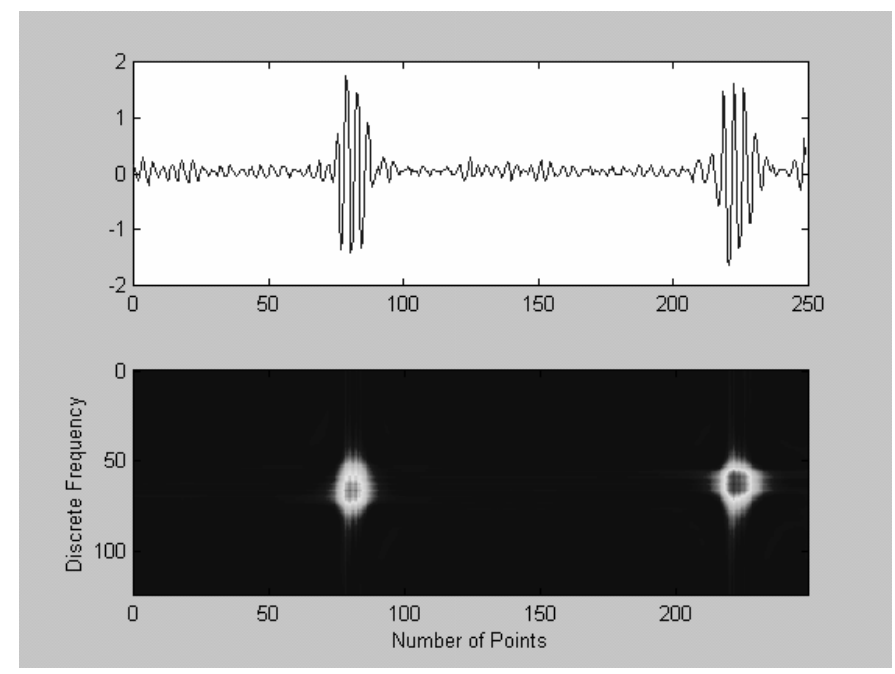

Figure 6.8 Echo-pulse signal processed using TFD with first flaw

Figs. 6.9 and 6.10, present similar information with respect to flaw 2 and flaw 3 , respectively. 


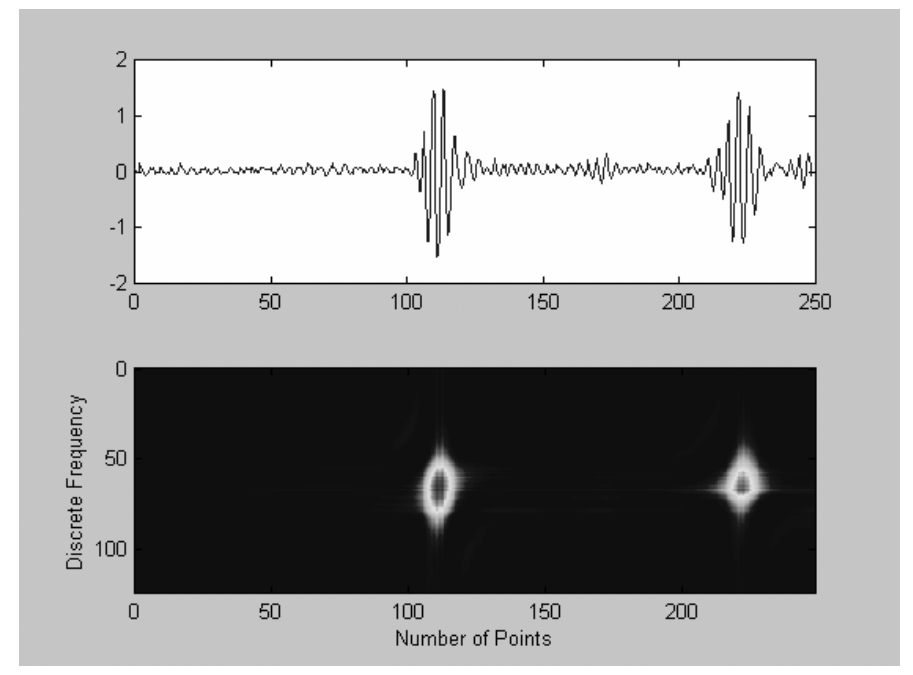

Figure 6.9 Echo-pulse signal processed using TFD with second flaw

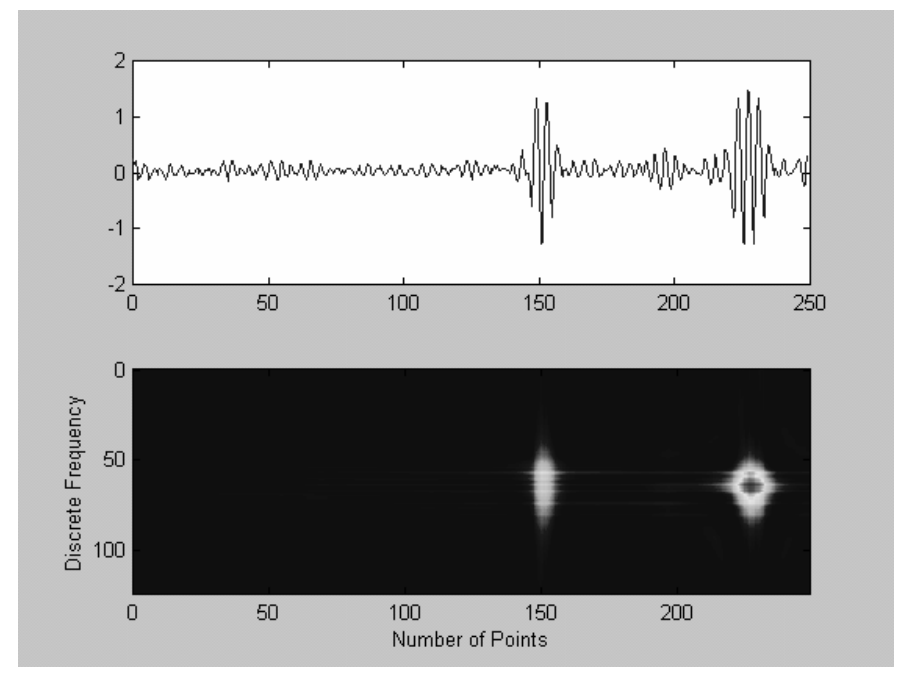

Figure 6.10 Echo-pulse signal processed using TFD with third flaw

The scenarios related to each flaw and the bottom parts are used to train the ART2 network. It is important to state that this training stage is not mixed with other TFD choices. The Bessel-ART2 algorithm processes the B-Scan information, which characterizes the sampled material. The neural network has been trained using the Bessel TFD. At the end of this procedure, it is a weight matrix that represents the B-scan of the sampled material. A transversal view of sampled material is constructed based upon the weight matrix of ART2 network. Fig. 6.11 depicts this information, where the y-axis presents the number of patterns, and the x-axis presents the temporal distance in terms of points. 


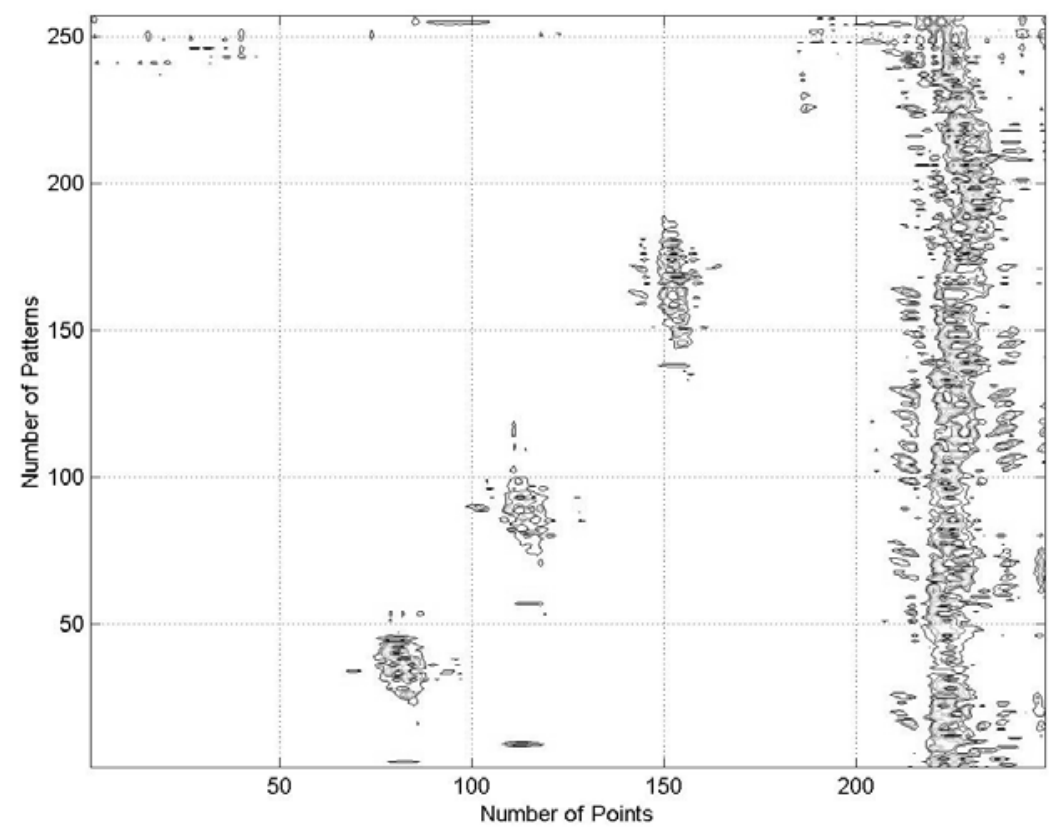

Figure 6.11 Transversal view of whole sampled material based upon the ART2 network approach

Fig. 6.12 shows one signal used to test the ART2 network without having been processed by the TFD module. In this case, one of the patterns (no. 95) has been selected. The number of patterns remains constant (250).
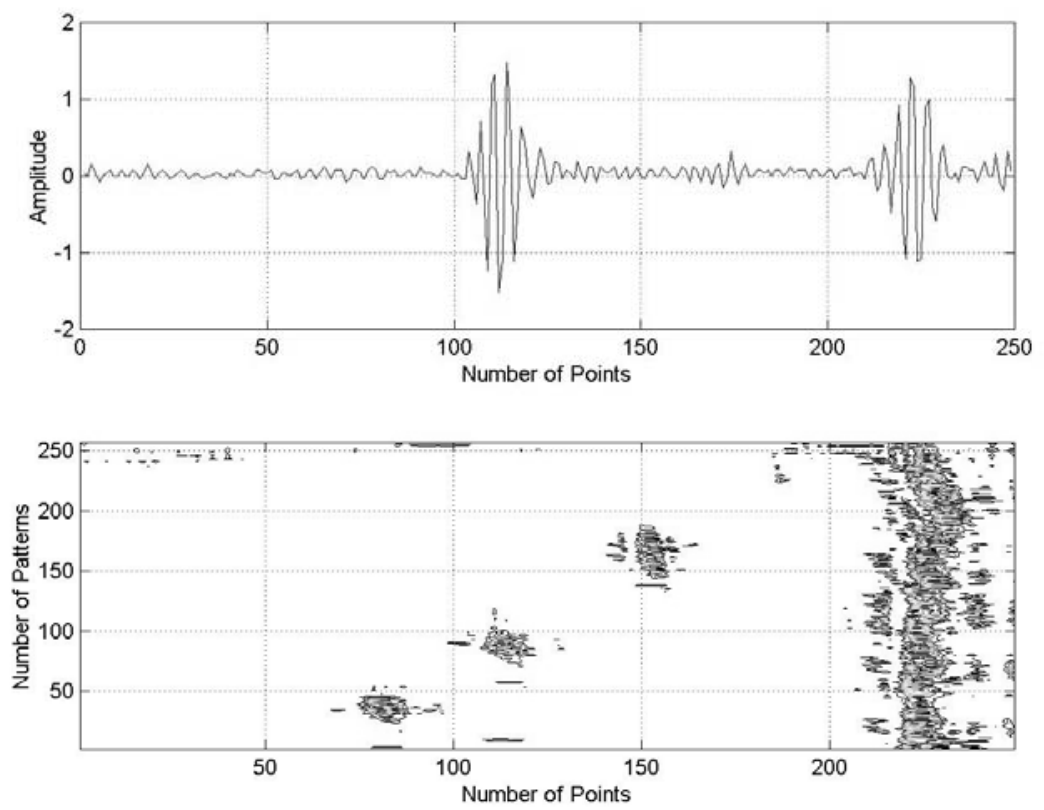

Figure 6.12 Evaluated database based upon a pulse-echo signal 
The last part of the experiment is presented in terms of the Choi-Williams TFD. In this case, pre-processing information is performed as for the Bessel TFD. The number of points taken into account is 499 . As presented in Wigner-Ville and Bessel experiments, all scenarios used to train the ART2 network are shown in Figs. 6.13, 6.14 and 6.15. Each of them represent first, second, and third flaw, respectively. For every case, half of the TFD response is presented due to the length of the sampled vector sub-sampled to fifty percent. This is possible because the central frequency of the transducer is similar to the sampling strategy. Therefore, no main information is lost. Nevertheless, this solution is ad-hoc to the used transducer; this approach allows a faster computation even though this was not the goal of the study.
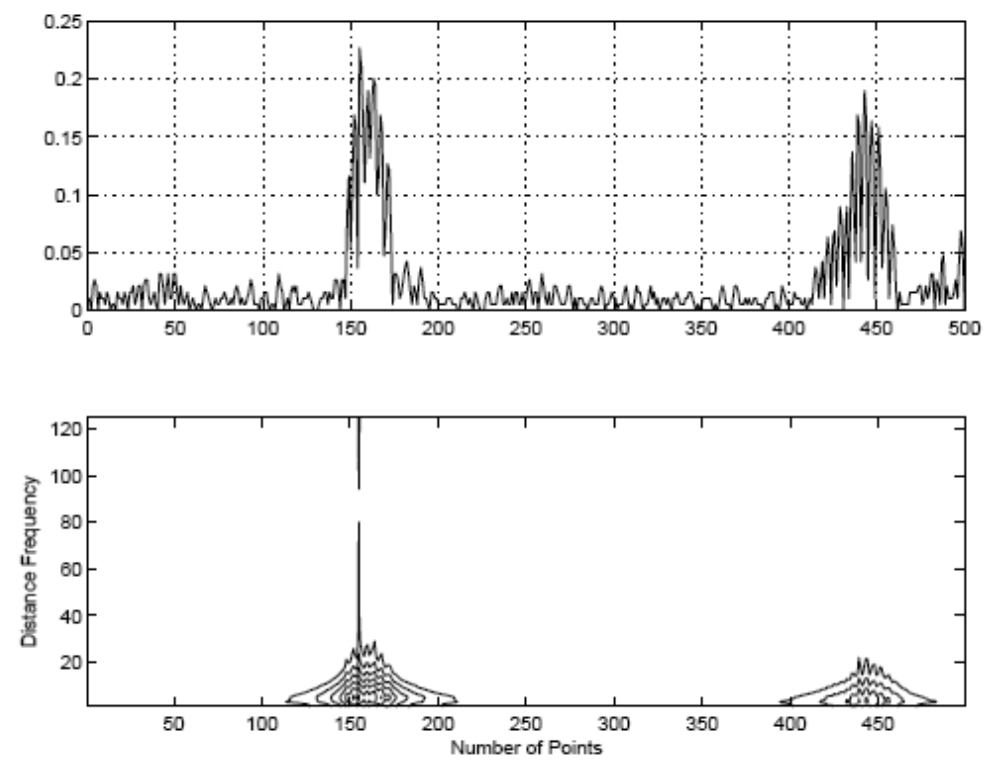

Figure 6.13 First flaw and echo-pulse signal processed using Choi-Williams TFD
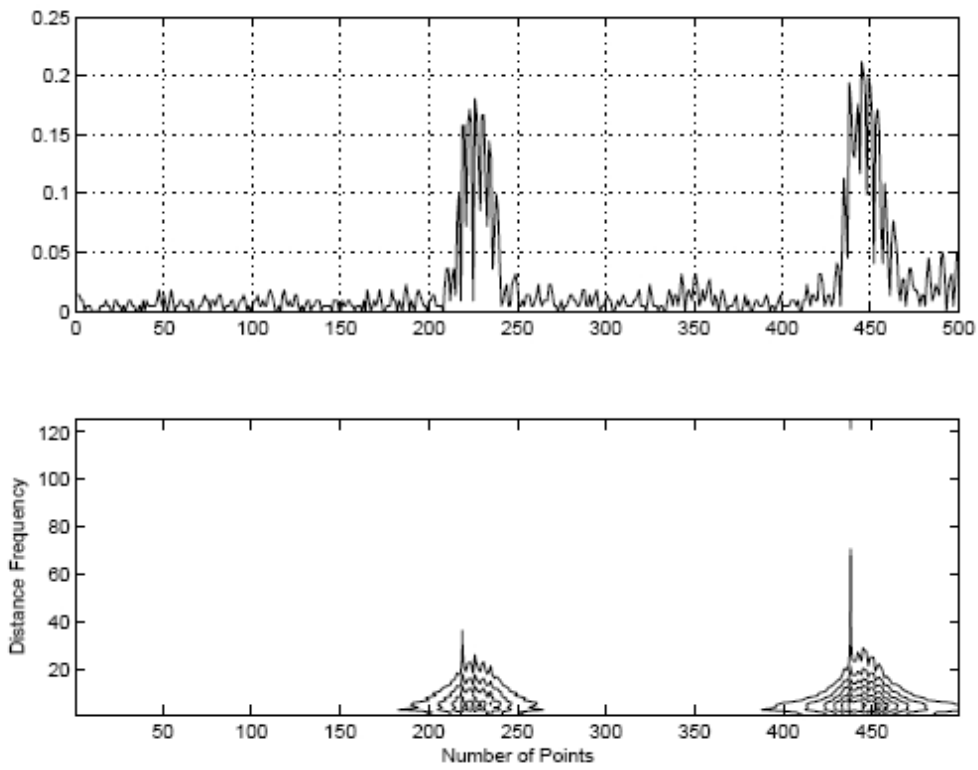

Figure 6.14 Second flaw and echo-pulse signal processed using Choi-Williams TFD 

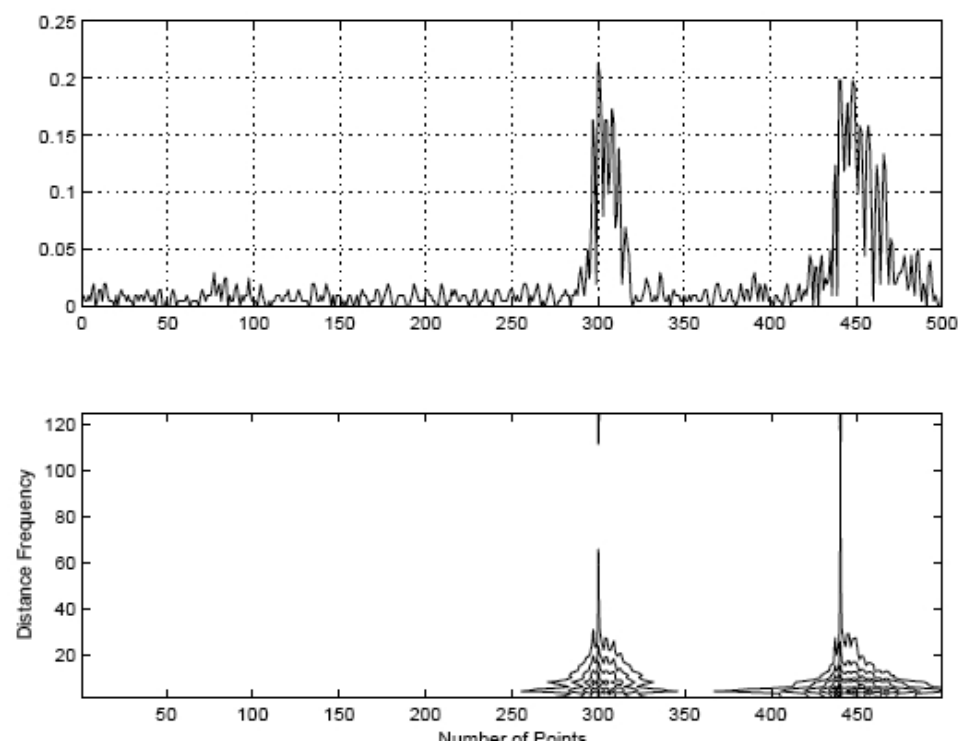

Figure 6.15 Second flaw and echo-pulse signal processed using Choi-Williams TFD

Integration of the pattern matrix is performed next with learning parameters of 0.3 and vigilance parameter of 0.95 . In this case, 140 patterns are classified as shown in Fig. 6.16.

As a result of these three evaluations, it has been found that Choi-Williams presents the best pattern selection in terms of number of the patterns and the quality of those patterns that depict the current flaws. It is important to mention that this matrix (Fig. 6.16) only shows a one-dimensional map of the flaws and the bottom part of the element. Therefore, the axes which depict the number of selected patterns are not related to the other lateral dimensions of the sampled material. This matrix has a similar form of the sampled material due to the fact that the neural network is performed following the B-Scan. Ten samples from the B-scan are chosen for training stage for all of the TFD used in the study.

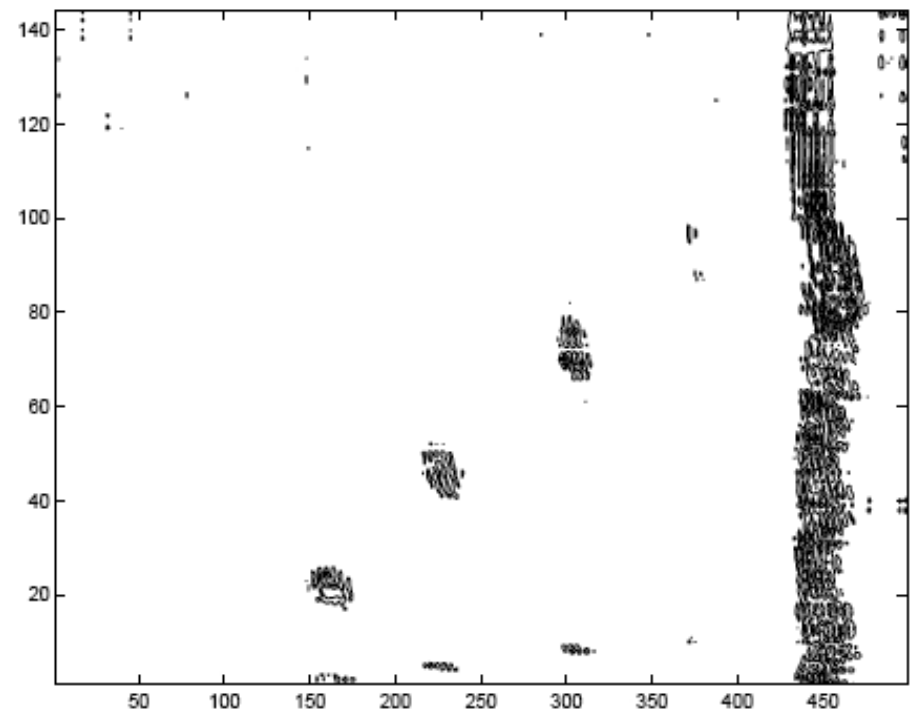

Figure 6.16 Transversal view of whole sampled material 


\section{CONCLUSIONS}

This work has shown the use of a Time frequency Distribution strategy combined with a neural network for Non-Destructive Evaluation. This strategy has shown to be an alternative approach for classification of flaws with no physical information from the current case study. Furthermore, pattern databases have been constructed based upon several selected echo-pulse signals, which have been obtained off-line.

This initial information is basic in order to obtain an accurate model of the inspected material. A key point of this strategy is the use of two algorithms, the TFD algorithms and the ART2 network - which are not separate algorithms with respect to the whole process. This approximation enhances the capabilities of the simple use of neural network for pattern classification. This strategy has shown an alternative approach for classification of abnormal situations with no information from current case study.

Furthermore, pattern databases have been constructed based upon several selected echo-pulse signals, which have been obtained off-line. This initial information is basic in order to obtain an accurate model of the inspected material.

Further work is required to justify the use of one specific Time Frequency Distribution approach over the rest of the current algorithms. Moreover, this strategy could address a proper dynamic non-linear system for on-line classification of unknown scenarios.

\section{ACKNOWLEDGMENTS}

The authors thank the financial support of PAPIIT-UNAM (IN106100 and IN105303), Mexico in connection with this work. Furthermore, the authors gratefully acknowledge fruitful discussions with $\mathrm{Dr}$. Arturo Juarez from CIATEQ, Mexico.

\section{REFERENCES}

[1] Angrisani, L., Daponte, P., and Dápuzzo, M., Wavelet Network-Based Detection and Classification of Transients, IEEE Transactions on Instrumentation and Measurement, 2001, 50, 1425-1435.

[2] Baraldi, A.,. and Blonda, P., A Survey pf Fuzzy Clustering Algorithms for Pattern Recognition-Part I, IEEE Transactions on Systems, Man and Cybernetics- Part B: Cybernetics, 1999, 29, 778-785.

[3] Benítez-Pérez H., Rubio-Acosta, E., Medina L. and García-Nocetti F., A Bessell Time Frequency Distribution and ART2 Network approach in Non Destructive Evaluation World Congress of Ultrasound; WCU, 2003 France, October.

[4] Carpenter, G. A., and Grossberg, S., ART2: Self organization of stable recognition codes for analog input patterns, Appl. Opt., 1987, 26, 23, 4919-4930.

[5] Ciftcioglu, Ö., From Neural to Wavelet Network, IEEE, NAFIPS, 18th International Conference of the North American, 1999, 894-898..

[6] Cohen L., Time-Frequency Distributions- A Review, Proceedings of the IEEE, 1989, 77, 941-981.

[7] Demirli, R., and Samiie, J., Model-Based Estimation of Ultrasonic Echoes Part II: Nondestructive Evaluation Applications, IEEE Transactions on Ultrasonics, Ferroelectrics, and Frequency Control, 2001a, 48, 3, 803-811..

[8] Demirli, R., and Samiie, J., Model-Based Estimation of Ultrasonic Echoes Part I: Analysis and Algorithms, IEEE Transactions on Ultrasonics, Ferroelectrics, and Frequency Control, 2001b, 48, 3, 787-802.

[9] Fang Y., and Chow T., Orthogonal Wavelet Neural Networks Applying to Identification of Wiener Model, IEEE Transactions on Circuits and Systems-I: Fundamental Theory and Applications, 2000, 47, 4, 591593. 
[10] Frank, T., Kraiss, K. F., and Kuhlen, T., Comparative Analysis of Fuzzy ART and ART-2A Network Clustering Performance, IEEE Transactions on Neural Networks, 1998, 9, 3, May.

[11] Frosberg F., Oung H., and Needleman L., Doppler Spectral Estimation Using Time-Frequency Distributions, IEEE Transactions on Ultrasonics, Ferroelectrics and Frequency Control, 1999, 46, 3.

[12] Garcia-Nocetti F., Solano-Gonzalez J., Rubio-Acosta E., and Moreno-Hernandez E., Fast Computation of Time Frequency Distributions using Parallel DSP-Based System for Signal Analysis, IFAC Conference on New Technologies for Computer Control, 2001, Hong Kong, 187-192.

[13] Kirby, M., Geometric Data Analysis, John Wiley and Sons, 2001, Canada.

[14] Legendre S., Goyette J., and , Massicotte D., Ultrasonic NDE of Composite material Structures using Wavelet Coefficients, NDT\&E International, 2001, 34, 31-37.

[15] Lester W. and Schmerr Jr., Fundamentals of Ultrasonic Nondestructive Evaluation, Plenum Press, 1998, USA.

[16] Martin W. and Flandrin P., Wigner-Ville Spectral Analysis of Nonstationary Processes, IEEE Transactions on Acoustics, Speech, and Signal Processing, 1985, ASSP-33., 6, 1461-1470.

[17] Mitra S., Digital Signal Processing, McGraw-Hill, 2001 USA.

[18] Moisen, M., H. Benítez, L. Medina, E. Moreno, G. González, and L. Leija, ARTMAP NETWORK AND WAVELET ANALYSIS FOR FLAWS CHARACTERIZATION, 16th World Conference on Nondestructive Testing, 2004, Toronto, Canada.

[19] Moya E., Sainz, G. I., Grande, B., Fuente M. J., and Perán J., Neural PCA Based Fault Diagnosis, Proceedings of the European Control Conference, 2001, 809-813, Oporto, Portugal.

[20] Oppenheim A., and Schafer R., Discrete-Time Signal Processing, Prentice Hall, 1999.

[21] Solís M., Benítez-Pérez H., Moreno E., Medina L., Gonzalez.G., Pattern Recognition of Wavelets Decomposition using ART2 Networks for echoes Analysis, IEEE International Ultrasonics Symposium, 2002, Munich, Germany, October.

[22] Solis, M.; Benitez-Perez, H.; Rubio, E.; Medina-Gomez, L.; Moreno-Hernandez, E., Pattern recognition of wavelets decomposition using ART2 networks for echoes analysis, Ultrasonics Symposium, 2001 IEEE, 2001, 1, 679-682.

[23] Tang Y., Dang, X., Liang, F., and Su, C., Dynamic Wavelet Network for Nonlinear Dynamic System Identification, Proceedings of the IEEE International Conference on Control Applications, 2000, 214219.

[24] Vachtsevanos, G., and Wang P., A Wavelet Neural Network Framework For Diagnostics of Complex Engineered Systems, IEEE, Proceedings of International Symposium of Intelligent Control, 2001, 7984, September.

[25] Whiteley, J., Davis, Mehrotra, A., and Ahalt, S., Observations and Problems Applying ART2 for Dynamic Sensor Pattern Interpretation, IEEE Transactions on Systems, Man, and Cybernetics- Part A: Systems and Humans, 1996, 26, 4, July.

[26] Yu, Y., and Tan, S., Vandewalle, J., and Deprettere E., Near-Optimal Construction of Wavelet Networks for Nonlinear System Modeling, IEEE International Symposium on Circuits and Systems, ISCAS, 1996, 3, $48-51$. 
Authors Biography

\section{Lucia Medina Gómez}

Earned her B. Sc degree in physical engineering from Universidad Autónoma MetropolitanaAzcapotzalco in1992, Mexico, and her Ph. D. degree in applied physics in 1998 from the University of Nottingham, Great Britain. There she worked in numerical analysis for ultrasonic 3D echo-location systems. She collaborated with the Institute of Applied Mathematics and Computational Systems, from 1999 to 2006 and now with the Physics Department of the Science Faculty, both at Universidad Nacional Autónoma de México. Her scientific interests are in nondestructive testing using ultrasound for characterization and flaws detection and location of composite materials

\section{Héctor Benítez Pérez}

Is a fulltime researcher at IIMAS UNAM (México). He obtained his first degree in electronic engineering in 1994 at UNAM. He obtained his Ph.D. at Sheffield University, UK in 1999. His research interests are in real-time control and fault diagnosis. 